VASCULAR BIOLOGY

\title{
A dark side to omega-3 fatty acids
}

The molecule 19,20-dihydroxydocosapentaenoic acid, formed by the metabolism of a fatty acid involved in normal brain function, promotes the development of a diabetes-associated form of blindness in a mouse model.

\section{KEISUKE YANAGIDA \& TIMOTHY HLA}

$\mathrm{D}$ iabetes can have many dangerous complications, including the progressive deterioration of blood vessels in the retina, which can lead to visual impairment. Despite the fact that this condition, called diabetic retinopathy, is a major cause of blindness in middle age, the pathways that mediate its development and progression are poorly understood. In a paper online in Nature, Hu et al. ${ }^{1}$ report that a derivative of the omega- 3 fatty acid docosahexaenoic acid (DHA) has a role in diabetic retinopathy.

Dietary intake of DHA is associated with several health benefits, including normal brain and eye function ${ }^{2}$. Moreover, mice that lack the protein Mfsd2a, which transports DHA across the blood-brain barrier into the brain, develop severe retinal and brain dysfunction $^{3-5}$. Omega-3 fatty acids such as DHA have key functions in cell membranes, where they provide membrane fluidity and flexibility, and promote the membranes' role as barriers, allowing the selective transport of molecules into and out of cells ${ }^{6}$.

In addition, omega- 3 fatty acids can have signalling roles. DHA is highly susceptible to oxidation, producing 'lipid mediator' molecules that can regulate cell-cell signalling - for instance, to orchestrate the return to normal conditions following an inflammatory reaction ${ }^{7}$. One class of lipid mediator produced by DHA oxidation is epoxide molecules. These metabolites are rapidly degraded by the enzyme soluble epoxide hydrolase (sEH) to form dihydroxy derivatives such as 19,20-dihydroxydocosapentaenoic acid $(19,20-D H D P)^{8}$. Indeed, $19,20-D H D P$ is the major product of DHA metabolism in the retina?.

Given that DHA and epoxide metabolites are associated with beneficial retinal effects, might sEH play a part in diabetic retinopathy by reducing the levels of these molecules? Hu et al. set out to examine this possibility using a mouse model of the disease. They found that increases in sEH expression in Müller glia cells (which support neuronal function in the retina) correlated with the onset of several early signs of diabetic retinopathy. Such changes included: the degeneration of pericyte cells, which wrap around and provide support for the endothelial cells that make up the blood-vessel wall; an increase in the number of acellular capillaries, which have abnormal transport and barrier properties; and leakage of plasma out of vessels into the surrounding retinal tissue. These defects eventually lead to poor circulation in the retina, abnormal and uncontrolled blood-vessel formation, retinal bleeding and visual impairment ${ }^{10}$.

Importantly, the authors demonstrated that pharmacological inhibition of $\mathrm{sEH}$ or genetic suppression of the gene encoding sEH prevented these pathological events, suggesting that sEH has a causal role in diabetic retinopathy. How might it exert these effects? Hu and colleagues showed that it is the production of 19,20-DHDP, rather than the reduction in DHA or epoxide molecules, that is the key trigger of vascular degeneration.

Next, the group found that the mechanism of pathogenesis involves interactions between three proteins - presenilin 1, vascular endothelial (VE)-cadherin and neural (N)-cadherin. The two cadherins are an essential part of protein complexes called adherens junctions, which anchor cell-cell adhesion sites on cell surfaces to the cell interior. VE-cadherin is found at junctions between pairs of endothelial cells, and $\mathrm{N}$-cadherin is located at junctions between endothelial cells and pericytes.

The authors showed that presenilin 1 and the cadherins associated with cholesterol in clusters called lipid rafts in cell membranes (Fig. 1a). However, increased levels of 19,20-DHDP altered the biophysical properties of membranes, leading to decreased interactions between presenilin 1 and cadherins in lipid rafts, presumably by changing the distribution of cholesterol ${ }^{9}$ (Fig. 1b). This, in turn, led to internalization of cadherins into the cell, disrupting adherens junctions. Ultimately, this process weakened cell-cell adhesion, causing pericyte 'dropout' from the blood vessel and breaching of the vascular barrier.

Together, $\mathrm{Hu}$ and colleagues' results indicate that 19,20-DHDP acts through a different mechanism from that of another player in retinopathy - vascular endothelial growth factor (VEGF). This protein is induced under
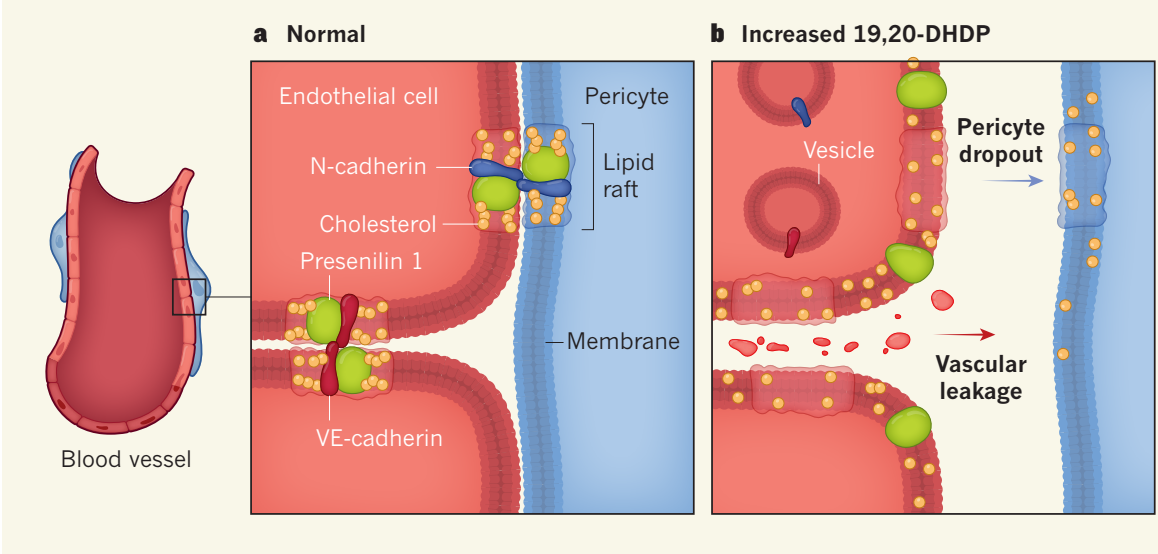

Figure 1 | Abnormal lipid dynamics in diabetic retinopathy. Hu et al. ${ }^{1}$ have shown that increases in levels of the molecule 19,20-DHDP promote the development and progression of a form of visual impairment called diabetic retinopathy in mice. a, Under normal conditions, the endothelial cells that line blood vessels in the retina are connected to one another and to supporting cells called pericytes through adhesion molecules known as cadherins in cell membranes. These connections rely on associations between the cadherins and the protein presenilin 1, which are, in turn, facilitated by clustering of cholesterol on 'lipid rafts'. b. The authors show that, during the early stages of diabetic retinopathy, increases in 19,20-DHDP in cell membranes (not shown) alter the distribution of presenilin 1, perhaps by disrupting the distribution of cholesterol molecules. Presenilin 1, no longer restrained to lipid rafts, does not associate with cadherins, leading to cadherin internalization in vesicles. This destabilizes cell-cell contacts, resulting in pericyte 'dropout' from around vessels, and vascular leakage. 
low-oxygen conditions and acts through receptors on vascular endothelial cells to induce proliferation, resulting in fragile new blood vessels prone to bleeding. Furthermore, the authors demonstrated that overexpression of sEH in the retinas of wild-type mice led to increased levels of 19,20-DHDP, vascular leakage and pericyte dropout, suggesting that $\mathrm{sEH}$ activation alone is sufficient to induce retinal-vessel defects, without VEGF-mediated mechanisms. Inhibiting VEGF halts the progression of diabetic retinopathy ${ }^{10}$ and that of another vision disorder caused by changes in retinal blood vessels: wet age-related macular degeneration ${ }^{11}$. But not all patients respond to anti-VEGF therapy, and those who do often become insensitive to treatment over time, making $\mathrm{Hu}$ and colleagues' discovery of an alternative pathway valuable.

Previous work ${ }^{12}$ has shown that a different lipid mediator that acts on endothelial cells, the molecule sphingosine 1-phosphate, induces the formation of adherens junctions and promotes the development of blood vessels. $\mathrm{Hu}$ and colleagues' newly discovered role for omega-3 derivatives thus adds to the list of crucial lipid signalling pathways known to be involved in retinal vascular diseases. It is possible that both pathways could be targeted therapeutically.
$\mathrm{Hu}$ and co-workers' study raises interesting avenues for future research. First, what triggers increases in $\mathrm{sEH}$ production in diabetic retinopathy? Dysregulated metabolic control of blood glucose can cause oxidative stress (an inability to deal with free radicals produced during metabolism) in the retina, which might be a key factor. However, a molecular mechanism by which this could lead to increased $\mathrm{sEH}$ remains to be elucidated. Second, it is unclear how 19,20-DHDP is transported from Müller glia cells to endothelial cells or pericytes. The apparent specificity of 19,20-DHDP among all omega-3 fatty acid derivatives as a mediator of diabetic retinopathy is also not understood at the molecular level. Perhaps 19,20-DHDP relies on specific targets, such as particular cellsurface or nuclear receptors, for its functions. Such targets could be a part of separate pathways to the membrane-lipid effects uncovered by the authors

In summary, $\mathrm{Hu}$ and colleagues' findings suggest that the metabolism of DHA to 19,20 -DHDP by sEH is one of the key regulatory systems that disrupt endothelial junctions at the blood-retinal barrier. The authors' work might help to guide the development of therapeutic approaches that block the activity of sEH to control diabetic retinopathy and wet age-related macular degeneration.
The study might even point to ways of treating neurological disorders, including stroke and neurodegenerative diseases, that are characterized by a compromised blood-brain barrier — similar mechanisms might be expected to operate in these diseases.

\section{Keisuke Yanagida and Timothy Hla} are in the Vascular Biology Program, Boston Children's Hospital, Department of Surgery, Harvard Medical School, Boston, Massachusetts 02115, USA. e-mail: timothy.hla@childrens.harvard.edu

1. Hu, J. et al. Nature http://dx.doi.org/10.1038/ nature25013 (2017).

2. Calder, P. C. Ann. Nutr. Metab. 69 (Suppl. 1), 8-21 (2016).

3. Nguyen, L. N. et al. Nature $\mathbf{5 0 9 , 5 0 3 - 5 0 6 ~ ( 2 0 1 4 ) . ~}$

4. Ben-Zvi, A. et al. Nature 509, 507-511 (2014).

5. Wong, B. H. et al. J. Biol. Chem. 291, 10501-10514 (2016).

6. Hishikawa, D., Valentine, W. J., lizuka-Hishikawa, Y., Shindou, H. \& Shimizu, T. FEBS Lett. 591, 2730-2744 (2017)

7. Serhan, C. N. Nature 510, 92-101 (2014).

8. Morisseau, C. \& Hammock, B. D. Annu. Rev. Pharmacol. Toxicol. 53, 37-58 (2013).

9. Hu, J. et al. J. Exp. Med. 211, 281-295 (2014).

10.Antonetti, D. A., Klein, R. \& Gardner, T. W. New Engl. J. Med. 366, 1227-1239 (2012)

11.Lim, L. S., Mitchell, P., Seddon, J. M., Holz, F. G. \& Wong, T. Y. Lancet 379, 1728-1738 (2012).

12.Paik, J.-H. et al. Genes Dev. 18, 2392-2403 (2004). 